\title{
Experimental study on synchronization of sound-coupled electronic metronomes
}

\author{
Hisashi Kada ${ }^{1}$, Kei Kondo ${ }^{1}$, Shota Sumino ${ }^{1}$, \\ and Isao T. Tokuda ${ }^{1 a}$ ) \\ ${ }^{1}$ Department of Mechanical Engineering, Ritsumeikan University, \\ 1-1-1 Noji-higashi, Kusatsu, Shiga 525-8577, Japan \\ a) isao@fc.ritsumei.ac.jp
}

Received April 12, 2016; Revised August 30, 2016; Published January 1, 2017

\begin{abstract}
As an experimental analogy of synchronized hands clapping (Néda et al., 2000), sound-coupled electronic metronomes are introduced. Clicking sounds generated from the driving metronome serve as the sounds of hands clapping, whereas a microphone attached to the driven electronic metronome detects the clicking sounds. In contrast to popular experimental systems of synchronization, e.g., mechanical metronomes put on a same beam, which directly connects oscillators through a material medium, the present system utilizes sound as an indirect coupling medium. In cases of both unidirectional and bidirectional couplings, our experiments showed that the two electronic metronomes are synchronized in such a way that a slow metronome catches up with a fast one. Phase slips, which give rise to intermittent switching between synchronized and desynchronized rhythms, were also observed, resembling the hands clapping in a concert hall. Our mathematical model based on phase oscillators with positive interaction function elucidates the observed results very well. Our system may provide a basic experimental framework for studying synchronization in sound-coupled oscillators including the rhythmic applause.
\end{abstract}

Key Words: synchronization, electronic metronomes, sound-coupling, phase oscillators, coupled oscillators

\section{Introduction}

Synchronization of coupled oscillators is an ubiquitous phenomenon in science and engineering [1-3]. Theoretical as well as experimental studies have been carried out on a variety of systems. Examples in nature include synchronization of firflies, entrainment of the circadian oscillators to environmental cycles [1], synchronization of circadian cells in the suprachiasmatic nucleus [4], synchronized walkers on the millennium bridge [5], and many others. Experimental systems range from mechanical systems (e.g., pendulums [6] and metronomes $[7,8])$, lasers $[9,10]$, electric circuits [11], acoustical systems (e.g., organ pipes [12]), biochemical systems to electro-chemical oscillators [13]. One of the most popular experimental systems to demonstrate synchronization in laboratories is a system of mechanical metronomes put on a same beam $[7,8,14]$. The coupling through the vibrating beam induces synchronized motion of the metronomes, i.e., they move always in a same (or opposite) di- 
rection. Complex dynamics including phase diffusions, overturnings, and hyperchaos have been also observed [8]. In contrast to such material medium, which directly connects the oscillators, there may exist an indirect coupling through another medium such as sounds. It has been reported that hand clapping becomes very well synchronized during audience applause in concert halls [15]. During an enthusiastic applause, the synchronized clapping can appear and disappear, where this process is repeated for several times. The synchrony of hands clapping has been well described by a phase oscillator model, where the repeated occurrence of synchrony and desynchrony has been considered to be due to change in a frequency detuning among natural frequencies of the individual hands clapping [16]. As an experimental analogy of the hand clapping, this paper studies synchronization of sound-coupled electronic metronomes. The clicking sounds generated from individual metronomes serve as the sounds of hands clapping, whereas microphone attached to the electronic metronome detects the clicking sounds. We experimentally show in two cases (both unidirectional and bidirectional couplings) that two electronic metronomes synchronize with each other, where a slow oscillator catches up with a fast one. On the borderline between synchrony and desynchrony in the parameter space, intermittent switchings between synchronized and desynchronized rhythms were observed, resembling the hands clapping in a concert hall. Our mathematical model based on phase oscillators with positive interaction function (excitatory coupling) elucidates the observed results very well. Our system may provide a basic experimental framework for studying synchronization in sound-coupled oscillators such as the synchronized hands clapping.

The rest of the paper is organized as follows. In Section 2, our experimental set-up for the soundcoupled electronic metronomes is introduced. In Section 3, experimental results on synchronized dynamics of the sound-coupled metronomes are reported. In Section 4, phase oscillator model is introduced to elucidate the mechanism of synchronization observed in the experimental system. The final Section is devoted for conclusions of our experiments and discussions on the future directions of the present study.

\section{Experimental system}

\subsection{Electronic metronome circuit}

Following the standard configuration $[17,18]$, each electronic metronome is based upon a circuit shown in Fig. 1. The core part is composed of PNP type transistor (Q1, 2SA1015, Toshiba), NPN type transistor (Q2, 2SC1815, Toshiba), resistor (R1, $10 \mathrm{k} \Omega$ ), potentiometer (VR1, SH16K4B502L20KC, Supertech Electronic), capacitor $(\mathrm{C} 1,100 \mu \mathrm{F})$, and diode (D1, 1SS270, Renesas Technology). The double transistor system is based on an astable multivibrator circuit, which operates as a relaxation oscillator [19]. The potentiometer (VR1) as well as the resistor (R1) is used to control the frequency range that the metronome operates over. Through another resistor $(\mathrm{R} 2,100 \mathrm{k} \Omega)$, an audio output is connected to the loudspeaker (SP, P-03285, Alpine). A microphone (AE-MICAMP) is inserted in parallel to the resistor (R1) so that the oscillation frequency of the metronome is affected by the input from the microphone.

\subsection{Experiment with unidirectional coupling}

Two electronic metronomes, which were constructed in the previous subsection, were unidirectionally coupled. A microphone was connected to one electronic metronome (called "transmitter"), whereas no microphone was connected to another (called "receiver"). As an observable of each metronome, the voltage of capacitor (C1) was measured by an oscilloscope (DSOX2014A, Keysight). As the forcing conditions, the following two parameters were considered.

(1) Frequency detuning, $\Delta f=f_{2}-f_{1}\left(f_{1}\right.$ : natural frequency of the receiver, $f_{2}$ : natural frequency of the transmitter).

(2) Distance, $d$, between the transmitter and the receiver.

To vary the frequency detuning, natural frequency of the transmitter $\left(f_{2}\right)$ was changed by adjusting its potentiometer (VR1), whereas that of the receiver $\left(f_{1}\right)$ was fixed throughout the experiment. To 


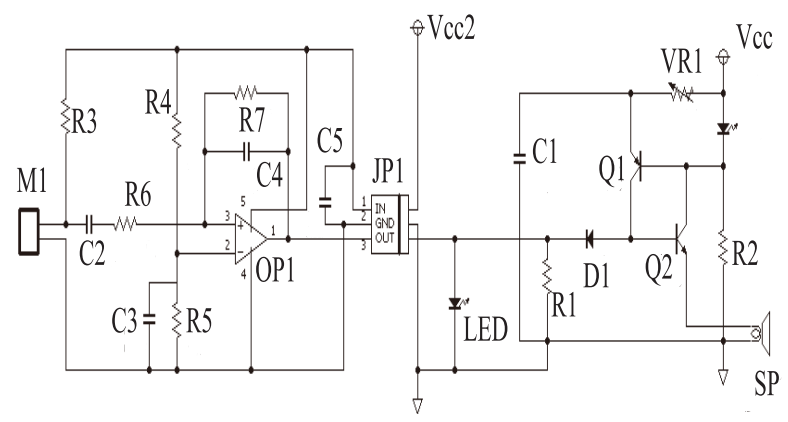

(a)

(b)

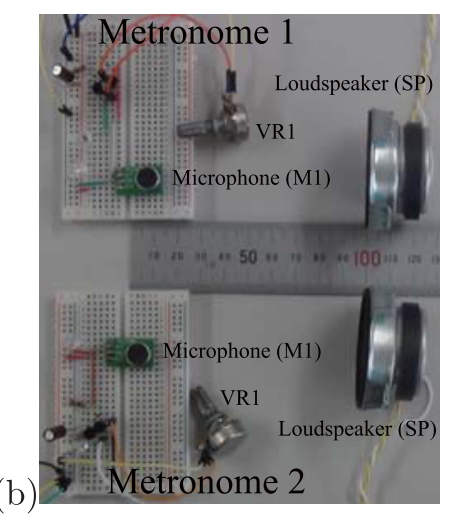

Fig. 1. (a) Circuit diagram of electronic metronome. The core part is composed of double transistors (PNP type: Q1, NPN type: Q2), resistor (R1, 10 $\mathrm{k} \Omega$ ), potentiometer (VR1), capacitor (C1), and diode (D1). Loudspeaker (SP) is connected through another resistor (R2). Microphone system (M1) is attached to the metronome in parallel to resistor (R1). (b) Experimental setup for coupled two electronic metronomes.

vary the distance, location of the receiver's microphone was changed, whereas that of the transmitter's loudspeaker stayed at the same position. In each experiment, their distance was measured by a ruler.

\subsection{Experiment with bidirectional coupling}

To realize bidirectional coupling, microphones were connected to both electronic metronomes (named as "metronome 1" and "metronome 2"). The distance between the two metronomes was fixed. As an adjustable parameter, the frequency detuning, $\Delta f=f_{2}-f_{1}$ was controlled. Namely, natural frequency of metronome $1\left(f_{1}\right)$ was changed by adjusting the potentiometer (VR1), whereas that of metronome $2\left(f_{2}\right)$ was fixed throughout the experiment.

\section{Experimental results}

\subsection{Experiment with unidirectional coupling}

For experiment with unidirectional coupling, natural frequency of the receiver was fixed to $f_{1}=5.75$ $\mathrm{Hz}$, whereas that of the transmitter $\left(f_{2}\right)$ was varied around $5.75 \mathrm{~Hz}$. Figure 2 shows dependence of the oscillation frequencies (both transmitter and receiver) on the frequency detuning. The panels (a)-(d) correspond to four settings of distances: $d=15 \mathrm{~cm}, d=10 \mathrm{~cm}, d=5 \mathrm{~cm}$, and $d=1 \mathrm{~cm}$. In each panel, a region exists, where the oscillation frequencies of transmitter and receiver coincide with each other. This represents a clear sign of synchronization. Comparing the panels (a)-(d), the range of synchronization becomes narrower, as the distance $d$ is increased. This can be due to the effect of coupling strength, which is weakened by the elongated distance. Figure 3 depicts the regime of synchronization in a two-dimensional parameter space $(\Delta f, d)$. Somewhat distorted Arnold tongue is discernible. One notable characteristic is its existence only in the region of positive frequency detuning $(\Delta f \geq 0)$. This implies that the receiver is entrained to the transmitter, only when the transmitter oscillation is faster than that of the receiver. 


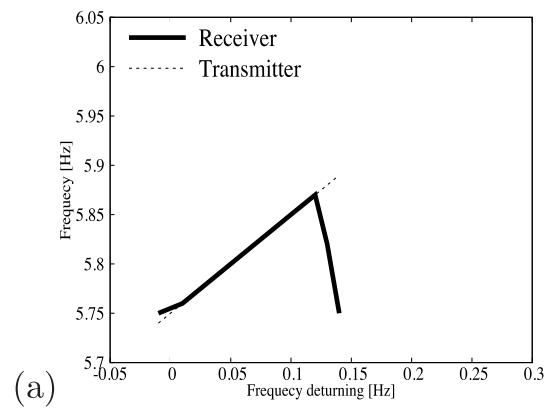

(a)

(b)

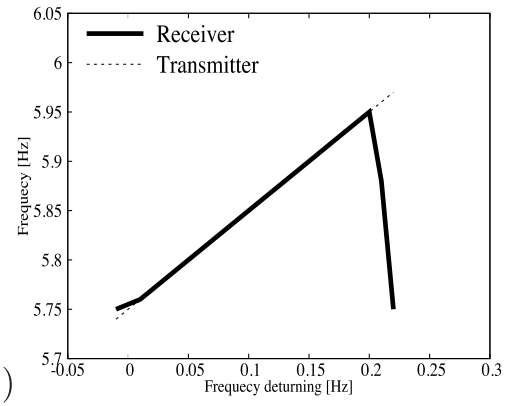

(c)
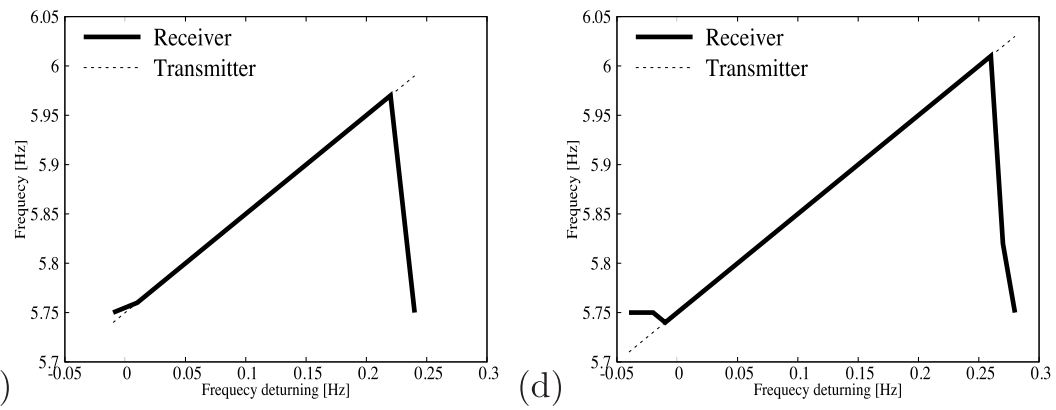

Fig. 2. Experiment with unidirectional coupling. Natural frequency of the receiver was fixed to $5.75 \mathrm{~Hz}$, whereas that of the transmitter was varied. Dependence of the oscillation frequencies (solid line: receiver, dotted line: transmitter) on the frequency detuning is shown. The distance between transmitter and receiver was (a) $d=15 \mathrm{~cm}$, (b) $d=10 \mathrm{~cm}$, (c) $d=5 \mathrm{~cm}$, and (d) $d=1$ $\mathrm{cm}$.

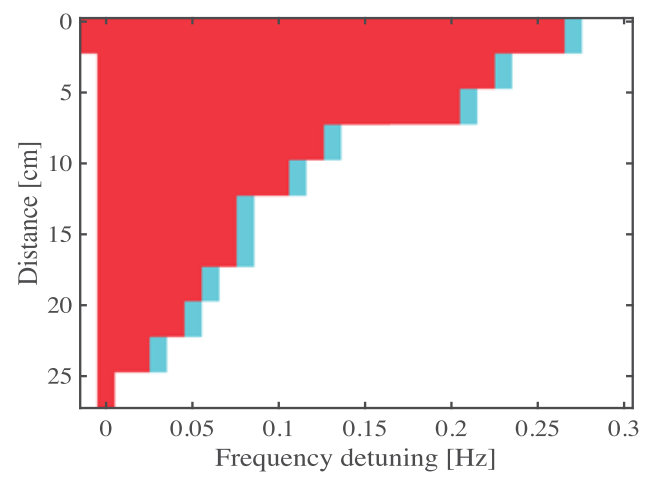

Fig. 3. Existence domain of synchronization in two-dimensional parameter space (abscissa: frequency detuning, ordinate: distance between transmitter and receiver). Between regimes of synchrony (red) and desynchrony (white), regions, in which synchrony and desynchrony occur repeatedly, are indicated in sky-blue.

On the border between synchrony and desynchrony, repeated occurrence of synchronized and desynchronized oscillations was observed. Figure 4 shows the case when the distance was set to $d=1 \mathrm{~cm}$ and the frequency detuning was set to $\Delta f=0.27 \mathrm{~Hz}$. In a time interval from $5 \mathrm{sec}$ to $8 \mathrm{sec}$ (panel (a)), the receiver was entrained to the transmitter. Namely, the waveforms of transmitter and receiver overlapped with each other (panel (a)). The Lissage plot was concentrated on the diagonal line (panel (b)), where the power spectra of transmitter and receiver indicated the same peak (panel (c)). In another time interval from $0 \mathrm{sec}$ to $1.9 \mathrm{sec}$, on the other hand, the receiver and transmitter were desynchronized with each other, where the two waveforms did not overlap with each other, the Lissage plot was deviated from the diagonal line, and the power spectra indicated different peaks (panels (d)-(f)).

To further examine the switching between synchrony and desynchrony observed in Fig. 4, phases of the receiver and transmitter $\left(\phi_{1}(t), \phi_{2}(t)\right)$ were extracted from the corresponding voltage data $\left(x_{1}(t), x_{2}(t)\right)$ by the Hilbert transform [20,21]. Time evolution of the phase difference between the transmitter and receiver, $\phi_{2}(t)-\phi_{1}(t)$, is shown in Fig. 5. The synchronized oscillations, characterized by time intervals in which the phase difference stays almost constant, are occasionally interrupted by 
(a)

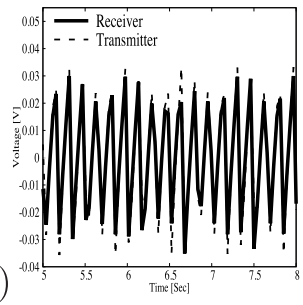

(d)

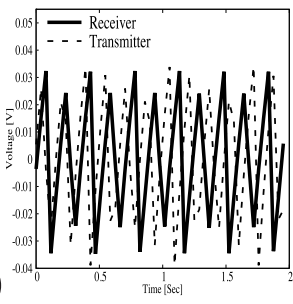

(b)

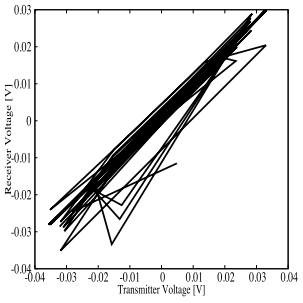

(e)

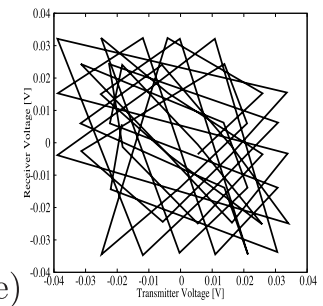

(c)

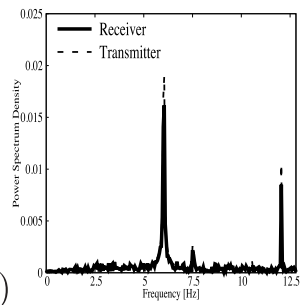

(f)

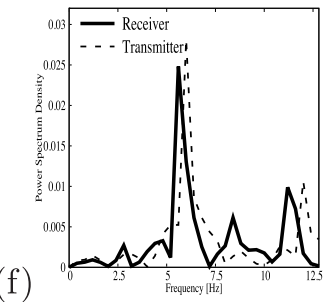

Fig. 4. Experiment with unidirectional coupling (distance: $d=1 \mathrm{~cm}$, frequency detuning: $0.27 \mathrm{~Hz})$. (a-c) correspond to data observed in time interval from $5 \mathrm{sec}$ to $8 \mathrm{sec}$, whereas $(\mathrm{d}-\mathrm{f})$ are observed in time interval from 0 sec to 1.9 sec. (a,d) Time-waveforms of transmitter (dotted) and receiver (solid). (b,e) Lissage plot (abscissa: transmitter, ordinate: receiver). (c,f) Power spectra of transmitter (dotted) and receiver (solid).

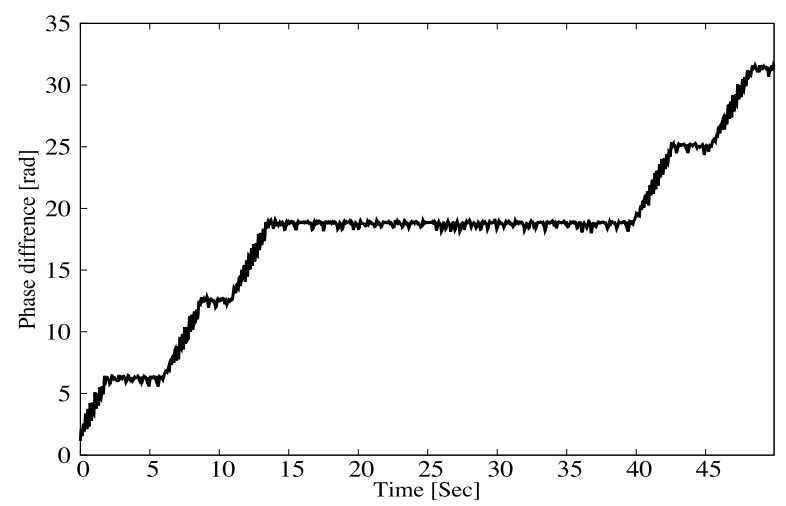

Fig. 5. Time evolution of the phase difference between transmitter and receiver, $\phi_{2}(t)-\phi_{1}(t)$, observed with unidirectional coupling (distance: $d=1$ $\mathrm{cm}$, frequency detuning: $0.27 \mathrm{~Hz}$ ).

phase jumps of one cycle $(2 \pi)$. This has been known as phase slips frequently observed on the border between synchronized and desynchronized regimes [3].

\subsection{Experiment with bidirectional coupling}

For experiment with bidirectional coupling, the distance between electronic metronomes 1 and 2 was set to $d=1 \mathrm{~cm}$. The natural frequency of metronome 1 was fixed to $f_{1}=5.53 \mathrm{~Hz}$ throughout the experiment. Figure 6 shows two cases, in which the natural frequency of metronome 2 was set to $f_{2}=5.42 \mathrm{~Hz}$ and $f_{2}=5.43 \mathrm{~Hz}$, respectively. In the former case, desynchronized oscillations are recognized, where the waveforms of the two metronomes did not overlap with each other, the Lissage plot was widely distributed, and the power spectra indicated slightly different peaks (panel (a)-(c)). In the latter case, in which the amount of the frequency detuning $|\Delta f|$ was made smaller, synchronized oscillations were observed. The two waveforms overlapped with each other, the Lissage plot was concentrated on the diagonal line, and the power spectra indicated the same peak (panel (d)-(f)).

Figure 7 shows dependence of the two oscillation frequencies on the frequency detuning, where the natural frequency of metronome 2 was varied from $f_{2}=5.25 \mathrm{~Hz}$ to $f_{2}=5.89 \mathrm{~Hz}$. A clear region of synchronization, in which the two oscillation frequencies coincide with each other, is recognized. Interestingly, the two oscillators were synchronized in such a way that a slow oscillator was accelerated to catch up with a fast oscillator. Namely, for frequency detuning ranged from $\Delta f=-0.1 \mathrm{~Hz}$ to $\Delta f=0 \mathrm{~Hz}$, frequency of the slow oscillator 2 was increased to $5.53 \mathrm{~Hz}$, i.e., natural frequency of 
(a)
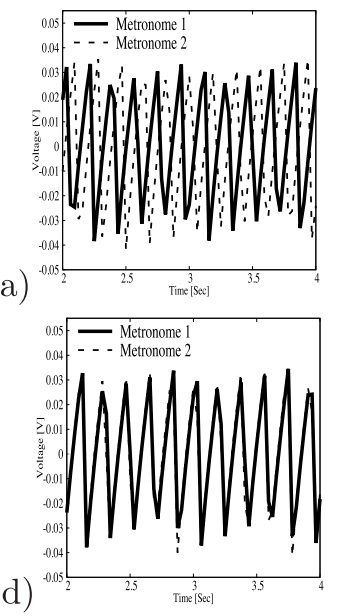

(b)
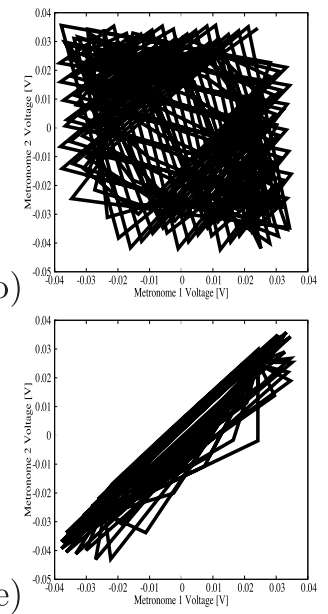

(c)
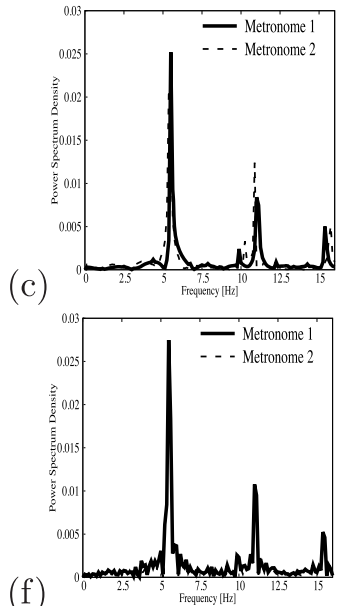

Fig. 6. Experiment with bidirectional coupling (distance: $d=1 \mathrm{~cm}$ ). The natural frequency of metronome 1 was fixed to $5.53 \mathrm{~Hz}$, whereas that of metronome 2 was set to $5.42 \mathrm{~Hz}$ in $(\mathrm{a}-\mathrm{c})$ and $5.43 \mathrm{~Hz}$ in $(\mathrm{d}-\mathrm{f})$. (a,d) Timewaveforms of metronomes 1 (solid) and 2 (dotted). (b,e) Lissage plot (abscissa: metronome 1, ordinate: metronome 2). (c,f) Power spectra of metronomes 1 (solid) and 2 (dotted).

the fast oscillator 1. For frequency detuning ranged from $\Delta f=0 \mathrm{~Hz}$ to $\Delta f=0.3 \mathrm{~Hz}$, frequency of the slow oscillator 1, whose natural frequency was set to $5.53 \mathrm{~Hz}$, was increased to that of the fast oscillator 2. This characteristic is essentially the same as that of the unidirectional coupling. It should be noted that there was a left-right asymmetry in the synchronization diagram (i.e., critical values for the frequency detuning were $\Delta f=-0.1 \mathrm{~Hz}$ and $\Delta f=0.3 \mathrm{~Hz}$ ). According to our measurement of the output voltages used to drive the loudspeakers (SP) in metronomes 1 and 2 , the output of metronome 2 was significantly larger than that of metronome 1 . Although the two metronomes have been constructed with the same architecture, certain difference in device properties as well as in setting of the variable resistor (VR1) may have caused such asymmetry. As a consequence, a louder sound was produced from the metronome 2 than from the metronome 1 . This implies that the forcing effect from metronome 2 to metronome 1 was larger than that from metronome 1 to metronome 2, creating the asymmetry in the synchronization diagram.

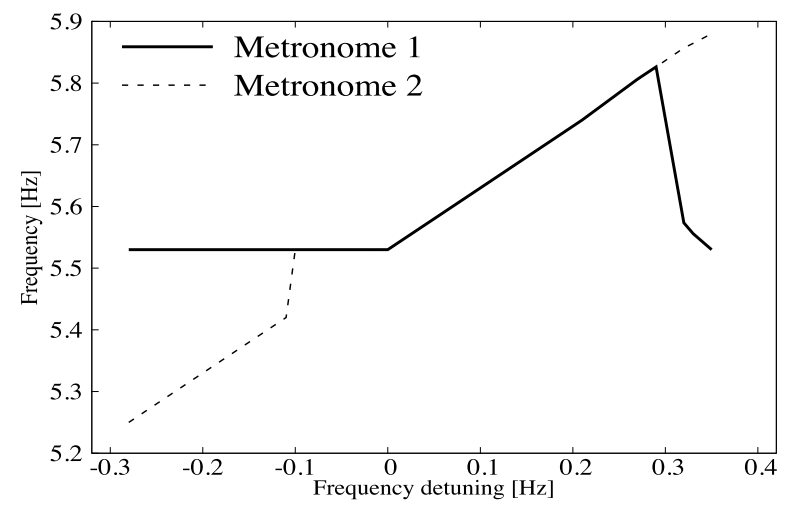

Fig. 7. Experiment with bidirectional coupling (distance: $d=1 \mathrm{~cm}$ ). The natural frequency of metronome 1 was fixed to $5.53 \mathrm{~Hz}$, whereas that of metronome 2 was varied from $5.25 \mathrm{~Hz}$ to $5.89 \mathrm{~Hz}$. Dependence of the frequencies (solid line: metronome 1, dotted line: metronome 2) on the frequency detuning is drawn.

In the case that the frequency detuning was $\Delta f=0.29 \mathrm{~Hz}$, which is on the border between regimes of synchrony and desynchrony, repeated occurrence of synchronized and desynchronized oscillations was observed. To display the phase relation between metronomes 1 and 2 , the corresponding phases $\left(\phi_{1}(t)\right.$, $\left.\phi_{2}(t)\right)$ were extracted by the Hilbert transform of the voltage data $\left(x_{1}(t), x_{2}(t)\right)$. Time evolution of the phase difference between the two metronomes, $\phi_{2}(t)-\phi_{1}(t)$, is shown in Fig. 8 . The phase slips are 


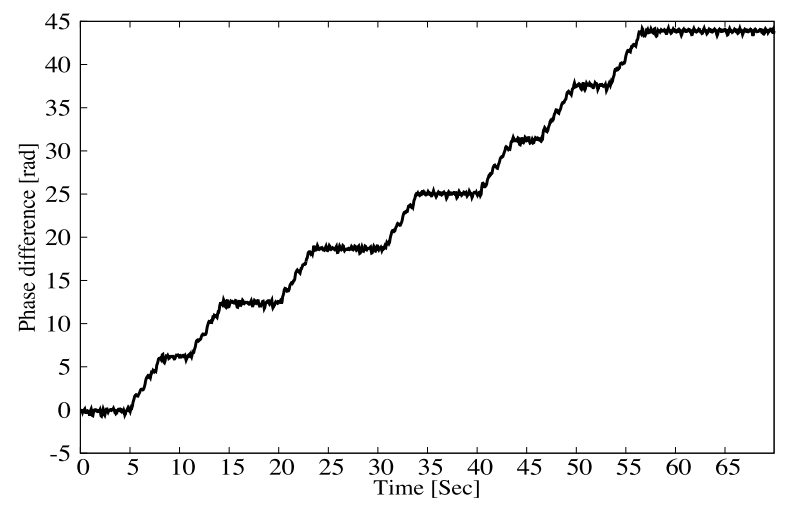

Fig. 8. Time evolution of the phase difference between bidirectionally coupled two metronomes, $\phi_{2}(t)-\phi_{1}(t)$ (distance: $d=1 \mathrm{~cm}$, frequency detuning: 0.27 $\mathrm{Hz})$.

observed as an underlying cause of the intermittent switching between synchrony and desynchrony. To view the experiments, demonstration videos are available for the synchronization [22] and phase slips [23] of the coupled electronic metronomes.

\section{Phase oscillator model}

To elucidate our experimental observations, equations of motion for the sound-coupled metronomes were constructed by the following phase oscillators [2]:

$$
\begin{aligned}
& \phi_{1}=\omega_{1}+T_{1}(d) H\left(\phi_{2}-\phi_{1}\right), \\
& \phi_{2}=\omega_{2}+T_{2}(d) H\left(\phi_{1}-\phi_{2}\right) .
\end{aligned}
$$

$\phi_{1}, \phi_{2}$ represent phases of the two electronic metronomes, $\omega_{1}, \omega_{2}$ represent their corresponding natural angular frequencies, $T_{1}, T_{2}$ stand for the coupling constants, and $H$ is the interaction function. As we have seen in the synchronization of coupled metronomes, a slow oscillator is speeded up to catch up with a fast oscillator. This implies that the coupling is of excitatory type, which increases the oscillation frequency. To realize such property, the interaction function, which is in general nonlinear and periodic with respect to $2 \pi$, was designed as $H(\Delta \phi)=\sin \Delta \phi$ if $\Delta \phi<\pi$ and $H(\Delta \phi)=0$ otherwise. A simple sine function without higher-order Fourier expansions was used because it provides a standard formula known to be able to capture the essence of synchronization in coupled oscillators [24]. With this interaction function, when the phase of slow oscillator is behind that of fast oscillator, oscillation frequency of the slow oscillator is increased by the positive (excitatory) coupling.

To imitate the experimental situation, the coupling strength $T$ was formulated as $T(d)=a \sigma\left(b / d^{2}\right)$, where $d$ represents the distance between two metronomes and $a$ and $b$ are constants. Since the sound from the loudspeaker spreads spherically, its intensity may decrease as $\sim d^{-2}$. A sigmoidal function $\sigma(x)=-1+2 /\left\{1+e^{-x}\right\}$ was used to describe the saturation effect of the microphone. The constant values were empirically determined as $a=1.5$ and $b=250$.

First, to simulate the experiment with unidirectional coupling, the coupling strength for the second oscillator was set to zero, i.e., $T_{2}=0$. The natural frequency of the first oscillator was fixed to 5.75 $\mathrm{Hz}$ (i.e., $\omega_{1}=2 \pi \cdot 5.75$ ), whereas that of the second oscillator was varied from $5.7 \mathrm{~Hz}$ to $6.05 \mathrm{~Hz}$ (i.e., $\left.\omega_{2} \in[2 \pi \cdot 5.7,2 \pi \cdot 6.05]\right)$. The distance was varied as $d \in[0 \mathrm{~cm}, 15 \mathrm{~cm}]$. Figure $9(\mathrm{a})$ shows the regime of synchronization computed by the phase oscillator model. The Arnold tongue qualitatively similar to the experiment was reproduced. As observed in the experiment, the synchronization exists only in the region of positive frequency detuning. This is due to the positive coupling implemented in the interaction function.

Next, the phase oscillator model was simulated to reproduce the experiment with bidirectional coupling. As described in Subsection 3.2, due to asymmetry between electronic metronomes 1 and 2 , the forcing effect from metronome 2 to metronome 1 seemed larger than that from metronome 1 to metronome 2. In other words, the coupling effect on the second oscillator seemed smaller than that 
on the first oscillator. To realize such asymmetric coupling, the interaction function of the second oscillator was set as $T_{2}(d)=0.5 \cdot T_{1}(d)$, where $T_{1}(d)$ is the same as that used in the unidirectional simulation and the ratio of 0.5 was empirically determined. The natural frequency of the first oscillator was fixed to $5.53 \mathrm{~Hz}$ (i.e., $\omega_{1}=2 \pi \cdot 5.53$ ), whereas that of the second oscillator was varied from 5.25 $\mathrm{Hz}$ to $5.89 \mathrm{~Hz}$ (i.e., $\left.\omega_{2} \in[2 \pi \cdot 5.25,2 \pi \cdot 5.88]\right)$. The distance was fixed to $d=1 \mathrm{~cm}$.

Figure 9(b) shows dependence of the two oscillation frequencies on the frequency detuning $\Delta f=$ $\left(\omega_{2}-\omega_{1}\right) /(2 \pi)$. For frequency detuning ranged from $\Delta f=-0.12 \mathrm{~Hz}$ to $\Delta f=0.24 \mathrm{~Hz}$, a regime of synchronization qualitatively similar to the experiment of Fig. 7 was reproduced. As observed in the experiment, the two oscillators were synchronized in such a way that frequency of the slow oscillator was increased to that of the fast oscillator. Again, this is due to the positive coupling implemented in the interaction function. As observed in the experiment, the regime of synchronization is wider on the side of positive detuning than that on the side of negative detuning. This imbalance is cased by the asymmetric interaction functions.

In both unidirectional and bidirectional couplings, the modeling curves reproduced qualitative properties of the experimental data in a satisfactory fashion. This implies that our experimental observation of the sound-coupled electronic metronomes can be primarily described by the phase oscillator model with positive interaction.

(a)

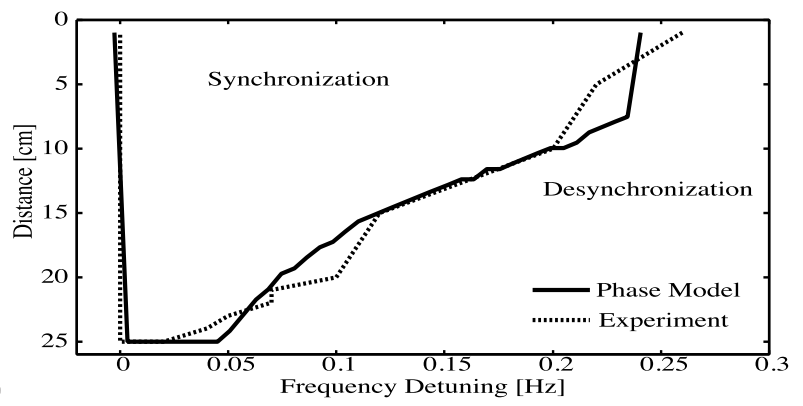

(b)

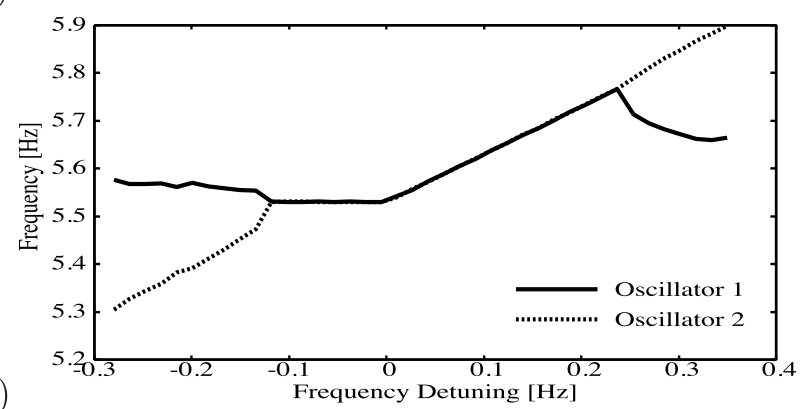

Fig. 9. Simulation results of the phase oscillator model. (a) Case of unidirectional coupling. Existence domain of synchronization is indicated in twodimensional parameter space (abscissa: frequency detuning, ordinate: distance between transmitter and receiver). The model curve (solid line) is compared with the experimental curve (dotted line). (b) Case of bidirectional coupling. Dependence of the frequencies (solid line: oscillator 1, dotted line: oscillator 2) on the frequency detuning.

\section{Discussions}

To summarize, we studied synchronization of the sound-coupled electronic metronomes as an analogy of hands clapping observed in concert halls [15]. Compared to archetypal experimental systems of coupled systems, which directly interact with each other through material medium, e.g., beam that couples mechanical metronomes [7], the present system utilizes sound as an indirect coupling medium. By adjusting the coupling strength (i.e., distance between two metronomes) as well as the frequency detuning, synchronized dynamics of the two metronomes was successfully observed in experiment with both unidirectional and bidirectional couplings. The key feature of the mechanism of synchronization is that an excitatory coupling increases the frequency of the slow oscillator so as to catch up with that 
of the fast oscillator. Indeed, the phase model, which implemented the excitatory coupling by the positive interaction function, reproduced the experimental phenomena fairly well. Our system may provide a basic experimental framework for studying synchronization in sound-coupled oscillators such as the rhythmic applause. The present framework might be also usefull as a simple yet instructive instance for demonstrating synchronization in laboratories or in classrooms [22, 23].

To compare the present results with the hands clapping in concert halls, several differences should be noted. First, it has been reported in hands clapping that the synchronization is observed when fast oscillators are slowed down to pace with slow oscillators $[15,16]$. This is opposite to what we have seen in our experiment. Second, during the synchronized applause, the audience feels more excitement and the speeds of their handclaps tend to be accelerated. As the individual frequencies of the handclaps are increased, they differ more from one to another and, as a consequence, distribution of the individual natural frequencies becomes more widely distributed. The frequency detuning, that is enlarged in this way, may finally destroy the synchrony [16]. Once the handclaps are desynchronized, the audience feels less excitement and their handclaps slow down. The decreased frequencies of the individual handclaps narrow the frequency distribution. With the reduced frequency detuning, synchronized hands clapping may reappear spontaneously. By this way, synchronized and desynchronized hands clapping appears and reappears repeatedly. In our experiment, on the other hand, the repeated occurrence of synchrony and desynchrony was due to phase slips, which are observed on the border of synchrony and desynchrony in a parameter space. In this case, natural frequencies of the oscillators do not change in time. This might be different from the hands clapping in concert halls, in which natural frequencies of the individual clapping are considered to change during the applause. To realize more analogous mechanism to the hands clapping, our future study may develop an inhibitory coupling that decreases the frequency of fast oscillator to get synchronized with slow oscillators. An additional mechanism, which increases natural oscillation frequencies when the metronomes are synchronized, should be also introduced.

Although one of our research interests has been in the effect of "indirect" coupling through sound on the synchronization phenomena, its effect was not significantly different from that of direct mechanical coupling reported in the preceding study [7]. To make the "indirect" effect more evident, the electronic metronomes should be located far apart from each other so that the sound propagation delay may introduce more complex dynamics such as repulsive interaction, phase shift, oscillation death, and chaos. To implement a more human-like auditory function, the microphone should have a selective filter that perceives sound input within a limited frequency range or a threshold that sets lower bound of the loudness to perceive sounds. Delays should be further introduced to the microphone, that transmits input stimuli to the metronome, so as to realize a human reaction time.

Finally, we note that the present work is limited by the experimental framework composed only of two oscillators. In the case of a network of coupled many oscillators, additional complexities such as clustering, spatio-temporal dynamics [2,3,24], phase diffusions, overturnings, and hyperchaos [8] should arise. As a model for hands clapping of audience, it is also essential to consider the population effect. A larger network of oscillators must be considered in our future study to realize the real situation of hands clapping of audience.

\section{Acknowledgments}

The authors would like to express their gratitude to Mr. Daiki Nagahama for his useful advise and technical assistance. They are also thankfull to the reviewers of the NOLTA journal for their critical reading of our manuscript. This study was partially supported by Grants-in-Aid for Scientific Research (No. 26286086, No. 16K00343).

\section{References}

[1] A.T. Winfree, The Geometry of Biological Time, Springer, New York, 1980.

[2] Y. Kuramoto, Chemical Oscillations, Waves and Turbulence, Springer, Berlin, 1984. 
[3] A.S. Pikovsky, M.G. Rosenblum, and J. Kurths, Synchronization: A Universal Concept in Nonlinear Sciences, Cambridge University Press, Cambridge, 2001.

[4] S. Yamaguchi, H. Isejima, T. Matsuo, R. Okura, K. Yagita, M. Kobayashi, and H. Okamura, "Synchronization of cellular clocks in the suprachiasmatic nucleus," Science, vol. 302, pp. 14081412, 2003.

[5] S.H. Strogatz, D.M. Abrams, A. McRobie, B. Eckhardt, and E. Ott, "Crowd synchrony on the Millennium bridge," Nature, vol. 438, no. 3, pp. 43-44, 2005.

[6] C. Hugenii, Horologium Oscillatorium. Apud F. Muguet, Parisiis, France, 1673.

[7] J. Pantaleone, "Synchronization of metronomes," Am. J. Phys, vol. 70, pp. 992-1000, 2002.

[8] H. Ulrichs, A. Mann, and U. Parlitz, "Synchronization and chaotic dynamics of coupled mechanical metronomes," Chaos, vol. 19, no. 4, p. 043120, 2009.

[9] A.E. Siegman. Lasers, University Science Books, Mill Valley, CA, 1986.

[10] J. Simonet, M. Warden, and E. Brun, "Locking and Arnold tongues in an infinite-dimensional system: The nuclear magnetic resonance laser with delayed feedback," Phys. Rev. E, vol. 50, pp. 3383-3391, 1994.

[11] L.M. Pecora and T.L. Carroll, "Synchronization in chaotic systems," Phys. Rev. Lett., vol. 64, pp. 821-824, 1990.

[12] M. Abel, S. Bergweiler, and R. Gerhard-Multhaupt, "Synchronization of organ pipes: experimental observations and modeling," J. Acoust. Soc. Am., vol. 119, no. 4, pp. 2467-2475, 2006.

[13] I.Z. Kiss, Y.M. Zhai, and J.L. Hudson, "Emerging coherence in a population of chemical oscillators," Science, vol. 296, pp. 1676-1678, 2002.

[14] http://www.youtube.com/user/IkeguchiLab?feature=watch

[15] Z. Néda, E. Ravasz, T. Vicsek, Y. Brechet, and A.-L. Barabási, "Self-organizing processes: The sound of many hands clapping," Nature, vol. 403, pp. 849-850, 2000.

[16] Z. Néda, E. Ravasz, T. Vicsek, Y. Brechet, and A.-L. Barabási, "Physics of the rhythmic applause," Phys. Rev. E, vol. 61, no. 6, pp. 6987-6992, 2000.

[17] L. Rodman, "Electronic transistorized metronome," U.S. Patent, vol. 3, no. 038, p. 120, June $5,1962$.

[18] R.L. Cannon, "Electronic metronome," U.S. Patent, vol. 4, no. 018, p. 131, April 19, 1977.

[19] A.A. Kumar, Fundamentals of Digital Circuits (2nd Revised), PHI Learning, 2009.

[20] D. Gabor, "Theory of communication," J. IEE (London), vol. 93, pp. 429-457, 1946.

[21] P. Panter, Modulation, Noise, and Spectral Analysis, McGraw-Hill, New York, 1965.

[22] https://www.youtube.com/watch?v=yGOZoXTAwI0

[23] https://www.youtube.com/watch?v=Qem7SazyevU

[24] J.A. Acebrón, L.L. Bonilla, C.J.P. Vicente, F. Ritort, and R. Spigler, "The Kuramoto model: A simple paradigm for synchronization phenomena," Reviews of modern physics, vol. 77, no. 1, pp. 137-185, 2005. 